\title{
Effects of Mental Flexibility and Motor Dysfunction on Cognitive Performance in Patients With Parkinson's Disease
}

\author{
Paraskevi Kourtidou ${ }^{1, *}$; Dimitrios Kasselimis ${ }^{1,2}$; Constantin Potagas ${ }^{1}$; Ioannis Zalonis ${ }^{1,3}$; \\ Ioannis Evdokimidis ${ }^{1}$ \\ ${ }_{1}^{1}$ First Department of Neurology, Aeginition Hospital, Medical School, National and Kapodistrian University, Athens, Greece \\ 2 Department of Psychology, University of Crete, Rethymno, Greece \\ ${ }^{3}$ Neuropsychological Laboratory, First Department of Neurology, Aeginition Hospital, Medical School, National and Kapodistrian University, Athens, Greece \\ ${ }^{*}$ Corresponding author: Paraskevi Kourtidou, First Department of Neurology, Aeginition Hospital, Medical School, National and Kapodistrian University, Athens, Greece. Tel: $+30-$ \\ 2107289307, Fax:+30-2107216474, E-mail: ekourtid@gmail.com
}

Received: June 10, 2014; Revised: October 8, 2014; Accepted: October 18, 2014

\begin{abstract}
Background: Parkinson's disease (PD) is a neurodegenerative disorder characterized by pervasive motor deficits, often accompanied by specific cognitive impairments, most prominently executive dysfunctions. However, it is often hard to determine whether the impaired performance of patients with PD on executive tests is the result of an executive dysfunction or whether it is due to motor difficulties.

Objectives: The present study aimed to examine the relationship between cognitive inflexibility, an executive dysfunction consistently found in PD, and motor deficiencies in patients with PD using derived scores, from a common executive function test named trail making test (TMT).

Patients and Methods: Fifty patients with PD and 50 demographically matched controls underwent neuropsychological examination which included TMT, part A and part B. Performance on each task was assessed and the following derived scores TMT B/A, TMT B-A/A, and TMT B-A were calculated and compared.

Results: Mann-Whitney tests revealed significant group differences for the mean TMTA $(\mathrm{P}=0.006)$ and TMT $\mathrm{B}(\mathrm{P}=0.000)$, which were both higher in the PD group, relative to controls. In addition, significant differences were found for one TMT product score (TMT B-A).

Conclusions: The results presented a significantly lower performance of patients with PD compared to controls, indicating the presence of a mental flexibility impairment which is dissociable and independent from the motor dysfunction.
\end{abstract}

Keywords:Parkinson's Disease; Trail Making Test; Executive Function

\section{Background}

Parkinson's disease (PD) is a neurodegenerative disease manifested by loss of dopamine-secreting neurons in the substantia nigra (1), leading to a well-established lack of dopamine in the striatum, disrupting the basal ganglia circuit (2). As a result, a series of functional changes that mediate the cardinal motor features of the disease (3), namely tremor, rigidity, akinesia/bradykinesia and postural deformities may emerge $(4,5)$. However, PD is not only a complex motor disorder but rather a systemic disease with various nonmotor deficits and neurological symptoms such as autonomic dysfunction (6), sensory (7) and sleep abnormalities (8), hallucinations (9) as well as neurobehavioral and cognitive disorders (10-12). Several comprehensive reviews have reported on impaired cognitive domains, including executive functions, visuospatial ability, speed of mental processing, memory, and learning $(10,11,13,14)$. Among the most common cognitive deficits associated with PD are those related to executive functions (15-17) and impaired mental flexibility appears to be the most prominent one $(15,18-20)$. Mental flexibility refers to the ability to shift between different thoughts and actions or between different aspects of a stimulus according to changes in a situation, utilize feedback, generate concepts, and devise alternative problem-solving strategies $(21,22)$. However, given the coexistence, as well as the positive relationship noted between poor cognitive performance and motor deficiencies in PD (23), slowed performance in executive tests could be the result of a variety of factors such as executive dysfunction (17), slowness in any stage in planning, initiating or executing motor responses (24), or perceptual analysis of a stimulus (25).

\section{Objectives}

The objective of the current study was to investigate whether the impaired performance of PD patients on executive tests is due to motor dysfunction, or due to an executive cognitive impairment such as mental rigidity. For this purpose we used Trail Making Test (TMT) Part A and B (26), which present the same motor and visuoperceptual demands, namely drawing lines to connect randomly arranged circles, while part $\mathrm{B}$ additionally measures divided

Copyright (C) 2015, Tehran University of Medical Sciences. This is an open-access article distributed under the terms of the Creative Commons Attribution-NonCommercial 4.0 International License (http://creativecommons.org/licenses/by-nc/4.0/) which permits copy and redistribute the material just in noncommercial usages, provided the original work is properly cited. 
attention and mental flexibility $(21,27,28)$. Since part B differs from part A only in terms of mental flexibility/divided attention, derived scores such as differences, ratios or proportional scores can be used to isolate it. Indeed, previous studies have made use of TMT derived scores, such TMT B-A, $\mathrm{B} / \mathrm{A}$ and (B-A)/A, in order to isolate mental flexibility or divided attention from performance (29). Among the three derived scores, TMT B-A is shown to be the most robust discriminator between PD patients and controls (30). Based on the above we attempted to test the hypothesis that performance of patients with PD on at least one TMT derived score namely TMT B-A would be significantly lower than expected, indicating that PD slowness is attributable to a mental flexibility deficit rather than a motor dysfunction.

\section{Patients and Methods}

\subsection{Participants}

Patients were recruited from the Neurological Clinic of Aeginition Hospital (Athens, Greece). Several inclusion criteria were implemented: (A) diagnosis of idiopathic PD, (B) classification below stage III (mild motor dysfunction) according to the Hoehn and Yahr scale (31) (C) mini mental state examination (MMSE) (32) score above 24, and (D) no psychiatric history. The PD group comprised 50 (14 females) right-handed patients, 32 to 81 years old (mean: 62.52, SD: 11.11), with 4 to 16 years of formal schooling (mean:11.50, SD: 4.06). The control group consisted of 50 neurologically intact, right-handed adults (17 females), matched for age, gender and educational level. Betweengroup differences on demographic characteristics were not significant, as revealed by t-tests for age and years of formal schooling as well as chi-square test for gender. Demographic characteristics of the two groups are shown in Table 1. Informed consent was obtained from each patient. Any human data included in this manuscript was obtained in compliance with regulations of the Aeginition Hospital ethics committee study, which conform to the ethical guidelines of the 1975 Declaration of Helsinki.

\subsection{Neuropsychological Examination}

TMT was administered to both PD and control groups by well-trained neuropsychologists, who were blind to the purposes of the study. Both parts (A and B) were administered according to the procedure described by Zalonis et al. (33), where the examiner points out errors as they occur, so that the patient can complete the test without errors and scoring is based on time alone (26). TMT is considered to measure scanning and visuomotor tracking, divided attention, and cognitive flexibility $(21,27)$. Two raw scores (time needed to complete TMT A and TMT B) and three derived scores (TMT B-A, TMT B/A, and TMT (BA)/A) were calculated for each participant.

\section{Results}

To test the normality of distributions, the one-sample Kolmogorov-Smirnov test was performed for each group, while skewness was also calculated. Since assumptions for normality were violated, nonparametric statistics were applied in subsequent analyses.Mann-Whitney tests showed significant group differences for both raw scores (patients with PD were slower). Regarding the derived scores, the two groups differed significantly only on TMT B-A. Performance of the PD group was lower for all the three derived scores compared to controls, but only the TMT B-A difference was significant. However, differences in the remaining two derived scores almost reached significance $(\mathrm{P}=0.054)$ (Table 2$)$.

\begin{tabular}{|c|c|c|c|c|c|}
\hline \multirow[t]{2}{*}{ Variable } & \multicolumn{2}{|c|}{ Parkinson's Disease } & \multicolumn{2}{|c|}{ Control } & \multirow[t]{2}{*}{ PValue } \\
\hline & Mean \pm SD & Range & Mean \pm SD & Range & \\
\hline Age, $y$ & $62.52 \pm 11.11$ & [32-81] & $62.42 \pm 11.17$ & [32-80] & $>0.05$ \\
\hline Education, $\mathbf{y}$ & $11.50 \pm 4.06$ & {$[4-16]$} & $11.70 \pm 3.56$ & {$[6-16]$} & $>0.05$ \\
\hline Gender, No. & & & & & $>0.05$ \\
\hline Male & 36 & & 33 & & \\
\hline Female & 14 & & 17 & & \\
\hline
\end{tabular}

Table 2. Performance of the Two Groups on Trail Making Test $(n=50)^{a}$

\begin{tabular}{|c|c|c|c|c|c|}
\hline \multirow[t]{2}{*}{ Variable } & \multicolumn{2}{|c|}{ PD Group } & \multicolumn{2}{|c|}{ Control Group } & \multirow[t]{2}{*}{ PValue } \\
\hline & Mean \pm SD & Range & Mean \pm SD & Range & \\
\hline TMT A, s & $71.62 \pm 31.19$ & $29-176$ & $60.18 \pm 29.48$ & {$[19-148]$} & $<0.05$ \\
\hline TMT B, s & $192.56 \pm 108.83$ & $42-512$ & $131.10 \pm 62.61$ & {$[45-276]$} & $<0.01$ \\
\hline Product score TMT B/A & $2.70 \pm 1.12$ & $1.28-6.53$ & $2.28 \pm 0.84$ & {$[1.11-5.19]$} & 0.054 \\
\hline Product score TMT B-A & $120.94 \pm 92.73$ & $13-412$ & $70.92 \pm 45.70$ & {$[8-185]$} & $<0.01$ \\
\hline $\begin{array}{l}\text { Product score TMT } \\
\text { (B-A)/A }\end{array}$ & $1.70 \pm 1.12$ & $0.28-5.53$ & $1.28 \pm 0.84$ & {$[0.11-4.19]$} & 0.054 \\
\hline
\end{tabular}

\footnotetext{
a Abbreviations: PD, Parkinson's disease; TMT, trail making test.
} 


\section{Discussion}

We expected that performance of patients with PD on TMT parts A and B would be significantly lower compared to controls. Indeed, patients with PD were slower than the matched controls in both parts of TMT, indicating reduced psychomotor speed and mental flexibility as well as divided attention. Previous studies have also demonstrated lower than expected performance of patients with PD on both TMT parts $(10,30,34)$.

However, raw TMT scores cannot differentiate between the effects of motor dysfunction and the mental rigidity observed in patients with PD, since, as previously mentioned, executive dysfunction can be coupled with and exacerbated by motor slowing $(35,36)$. Derived TMT scores have been used by various PD studies to subtract the effects of motor and psychomotor slowness from performance and as a more-sensitive discriminator between patients with PD and controls $(30,37,38)$. It is proposed that TMT B-A derived score is the most robust cognitive discriminator between patients and controls with a performance continuum through controls, patients with PD, and demented patients with PD (30). In our study, TMT B-A scores were significantly different between the two groups, thus indicating dissociation between motor dysfunction and mental rigidity of the patients with PD, regardless of the motor dysfunction.

TMT B/A also appears to be a sensitive indicator of cognitive decline in some cases $(29,38)$. Felmingham et al. (39) also recommended the use of a ratio score (TMT B/ TMT A), because slower performance on TMT-A appeared to underlie deficits in TMT-B. Slowing of information processing speed appears to be responsible for difficulties in more complex cognitive tasks. Our results did not show such differences in TMT B/A, although significance was almost reached $(P=0.054)$. It could be speculated that a greater sample could allow t-test analyses and reveal the expected differences in TMT B/A between patients with PD and controls.

In the present study, we stated the hypothesis that performance of patients with PD on at least one TMT-derived score namely TMT B-A would be significantly lower than expected, indicating that PD slowness is attributable to a mental flexibility deficit rather than a motor dysfunction. This was confirmed by the results. Overall, our findings suggest that lower performance on TMT B is not caused solely by a slowed motor performance, but is rather the result of a decreased ability to shift between sets; a process that is considered as a critical composite of mental flexibility $(21,40)$.

Even though our results support the existence of a mental inflexibility in the PD group, they do not exclude a possible motor and/or cognitive slowing co-existence. Indeed, since raw TMT-A scores show an impaired performance of patients with PD compared to controls, the overall lower performance of the PD group on TMT could be partly due to motor response difficulties or central processing speed deficits in the sense of a general cognitive slowing, besides mental flexibility deficiencies.

Our findings also suggest that motor slowness and cognitive dysfunction in terms of mental inflexibility can be dissociated based on TMT performance. These two different components have been suggested to involve different pathological and neural mechanisms. Therefore, subthalamic nucleus deep brain stimulation seems to improve motor function, but is often correlated with cognitive decline in domains such as executive function (41). Additionally, unilateral pallidotomy has been shown to increase contralateral upper limb mobility, but has had no effect on executive function performance, as measured by Stroop and TMT A and B (42). Furthermore, evidence from dopaminergic therapy has indicated that although dopamine replacement improves motor symptoms, some cognitive functions are improved, while others are unaltered or even hindered (43). The above findings indicate possible clinical significance of the use of TMT and its derived scores for assessing the effects of treatment on motor and cognitive function.

In conclusion, the performance of patients with PD on both parts of the TMT indicates that motor dysfunction and mental inflexibility are two distinct PD deficits which may coexist. However, the use of TMT test as well as the derived scores can help differentiate between their effects, providing a chance to evaluate the course of the disease while assessing the effects of treatment on motor and cognitive function. Future studies including a wide variety of cognitive measures could provide a better evaluation of a possible cognitive slowing, which may interfere with motor dysfunction and mental flexibility.

\section{References}

1. Davie CA. A review of Parkinson's disease. $\mathrm{Br}$ Med Bull. 2008;86:109-27.

2. Obeso JA, Rodriguez-Oroz MC, Benitez-Temino B, Blesa FJ, Guridi J, Marin C, et al. Functional organization of the basal ganglia: therapeutic implications for Parkinson's disease. Mov Disord. 2008;23 Suppl 3:S548-59.

3. Hirsch EC, Perier C, Orieux G, Francois C, Feger J, Yelnik J, et al. Metabolic effects of nigrostriatal denervation in basal ganglia. Trends Neurosci. 2000;23(10 Suppl):S78-85.

4. Berardelli A, Rothwell JC, Thompson PD, Hallett M. Pathophysiology of bradykinesia in Parkinson's disease. Brain. 2001;124(Pt 11):2131-46.

5. Jankovic J. Parkinson's disease: clinical features and diagnosis. $J$ Neurol Neurosurg Psychiatry. 2008;79(4):368-76.

6. Goetz CG, Lutge W, Tanner CM. Autonomic dysfunction in Parkinson's disease. Neurology. 1986;36(1):73-5.

7. Nolano M, Provitera V, Estraneo A, Selim MM, Caporaso G, Stancanelli A, et al. Sensory deficit in Parkinson's disease: evidence of a cutaneous denervation. Brain. 2008;131(Pt 7):1903-11.

8. Kumar S, Bhatia M, Behari M. Sleep disorders in Parkinson's disease. Mov Disord. 2002;17(4):775-81.

9. Goetz CG, Ouyang B, Negron A, Stebbins GT. Hallucinations and sleep disorders in PD: ten-year prospective longitudinal study. Neurology. 2010;75(20):1773-9.

10. Dubois B, Pillon B. Cognitive deficits in Parkinson's disease.J Neurol.1997;244(1):2-8.

11. Galvin JE. Cognitive change in Parkinson disease. Alzheimer Dis Assoc Disord. 2006;20(4):302-10. 
12. Koerts J, Leenders KL, Brouwer WH. Cognitive dysfunction in non-demented Parkinson's disease patients: controlled and automatic behavior. Cortex. 2009;45(8):922-9.

13. Cooper JA, Sagar HJ, Jordan N, Harvey NS, Sullivan EV. Cognitive impairment in early, untreated Parkinson's disease and its relationship to motor disability. Brain. 1991;114 ( Pt 5):2095-122.

14. Zgaljardic DJ, Borod JC, Foldi NS, Mattis P. A review of the cognitive and behavioral sequelae of Parkinson's disease: relationship to frontostriatal circuitry. Cogn Behav Neurol. 2003;16(4):193-210.

15. Cools R, Barker RA, Sahakian BJ, Robbins TW. Mechanisms of cognitive set flexibility in Parkinson's disease. Brain. 2001;124(Pt 12):2503-12.

16. Levy G, Jacobs DM, Tang MX, Cote LJ, Louis ED, Alfaro B, et al. Memory and executive function impairment predict dementia in Parkinson's disease. Mov Disord. 2002;17(6):1221-6.

17. McKinlay A, Grace RC, Dalrymple-Alford JC, Roger D. Characteristics of executive function impairment in Parkinson's disease patients without dementia. J Int Neuropsychol Soc. 2010;16(2):268-77.

18. Bowen FP, Kamienny RS, Burns MM, Yahr M. Parkinsonism: effects of levodopa treatment on concept formation. Neurology. 1975;25(8):701-4.

19. Caltagirone C, Carlesimo A, Nocentini U, Vicari S. Defective concept formation in parkinsonians is independent from mental deterioration. J Neurol Neurosurg Psychiatry. 1989;52(3):334-7.

20. Lees AJ, Smith E. Cognitive deficits in the early stages of Parkinson's disease. Brain. 1983;106 (Pt 2):257-70.

21. Lezak MD, Howieson DB, Loring DW. Neuropsychological Assessment.New York, USA: Oxford University Press; 2004.

22. LuriaAR.TheWorkingBrain:AnIntroductionTo Neuropsychology.New York, USA: Basic Books; 1976.

23. Verbaan D, Marinus J, Visser M, van Rooden SM, Stiggelbout AM, Middelkoop HA, et al. Cognitive impairment in Parkinson's disease. J Neurol Neurosurg Psychiatry. 2007;78(11):1182-7.

24. Brown RG, Marsden CD. Internal versus external cues and the control of attention in Parkinson's disease. Brain. 1988;111 ( Pt 2):323-45.

25. Revonsuo A, Portin R, Koivikko L, Rinne JO, Rinne UK. Slowing of information processing in Parkinson's disease. Brain Cogn. 1993;21(1):87-110.

26. Reitan R. Validity of the Trail Making Test as an indicator of organic brain damage. Percept Mot Skills. 1958;8:271-6.

27. Olivera-Souza RD, Moll J, Passman LJ, Cunha FC, Paes F, Adriano MV, et al. Trail making and cognitive set-shifting. Arq Neuropsiquiatr. 2000;58(3B):826-9.

28. Strauss E, Sherman EMS, Spreen O.A Compendium of Neuropsychological Tests: Administration, Norms, and Commentary. 3rd edNew York, USA: Oxford University Press; 2006.
29. Lamberty G, Putnam S. Derived Trail Making Test indices: A preliminary report. Neuropsychiatry Neuropsychol Behav Neurol. 1994;7(3):230-4.

30. Gasparini M, Bonifati V, Fabrizio E, Fabbrini G, Brusa L, Lenzi GL, et al. Frontal lobe dysfunction in essential tremor: a preliminary study.J Neurol. 2001;248(5):399-402.

31. Hoehn MM, Yahr MD. Parkinsonism: onset, progression and mortality. Neurology. 1967;17(5):427-42.

32. Fountoulakis KN, Tsolaki M, Chantzi H, Kazis A. Mini mental state examination (MMSE): a validation study in Greece. Am J Alzheimers Dis Other Demen. 2000;15(6):342-5.

33. Zalonis I, Kararizou E, Triantafyllou NI, Kapaki E, Papageorgiou S, Sgouropoulos D, et al. A normative study of the Trail Making Test A and B in Greek adults. Clin Neuropsychol. 2008;22:842-50.

34. Zamarian L, Visani P, Delazer M, Seppi K, Mair KJ, Diem A, et al. Parkinson's disease and arithmetics: the role of executive functions. J Neurol Sci. 2006;248(1-2):124-30.

35. Weintraub D, Moberg PJ, Culbertson WC, Duda JE, Katz IR, Stern MB. Dimensions of executive function in Parkinson's disease. Dement Geriatr Cogn Disord. 2005;20(2-3):140-4.

36. de Frias CM, Dixon RA, Fisher N, Camicioli R. Intraindividual variability in neurocognitive speed: a comparison of Parkinson's disease and normal older adults. Neuropsychologia. 2007;45(11):2499-507.

37. Bohnen NI, Kaufer DI, Hendrickson R, Ivanco LS, Lopresti BJ, Constantine GM, et al. Cognitive correlates of cortical cholinergic denervation in Parkinson's disease and parkinsonian dementia. J Neurol. 2006;253(2):242-7.

38. Kanazawa A, Mizuno Y, Narabayashi H. [Executive function in Parkinson's disease]. Rinsho Shinkeigaku. 2001;41(4-5):167-72.

39. Felmingham KL, Baguley IJ, Green AM. Effects of diffuse axonal injury on speed of information processing following severe traumatic brain injury. Neuropsychology. 2004;18(3):564-71.

40. Anderson V, Jacobs R, Anderson PJ. Executive Functions and the Frontal Lobes: A Lifespan Perspective (Studies on Neuropsychology, Neurology and Cognition).London: Psychology Press; 2008.

41. Halpern CH, Rick JH, Danish SF, Grossman M, Baltuch GH. Cognition following bilateral deep brain stimulation surgery of the subthalamic nucleus for Parkinson's disease. Int J Geriatr Psychiatry. 2009;24(5):443-51.

42. Olzak M, Laskowska I, Jelonek J, Michalak M, Szolna A, Gryz J, et al. Psychomotor and executive functioning after unilateral posteroventral pallidotomy in patients with Parkinson's disease. J Neurol Sci. 2006;248(1-2):97-103.

43. Macdonald PA, Monchi O. Differential effects of dopaminergic therapies on dorsal and ventral striatum in Parkinson's disease: implications for cognitive function. Parkinsons Dis. 2011;2011:572743. 